\title{
Atom-by-Atom STEM Investigation of Defect Engineering in Graphene
}

\author{
Q.M. Ramasse ${ }^{1}$, D.M. Kepapstoglou ${ }^{1}$, F.S. Hage ${ }^{1}$, T. Susi ${ }^{2}$, J. Kotakoski ${ }^{2,3}$, C. Mangler ${ }^{2}$, P. Ayala ${ }^{2}$, J.
} Meyer ${ }^{2}$, J.A. Hinks ${ }^{4}$, S. Donnelly ${ }^{4}$, R. Zan ${ }^{5}$, C.T. Pan ${ }^{5}$, S.J. Haigh ${ }^{5}$ and U. Bangert ${ }^{5,6}$

1 SuperSTEM Laboratory, STFC Daresbury Campus, Keckwick Lane, Daresbury WA4 4AD, U.K.

2 Faculty of Physics, University of Vienna, Strulhofgasse 4, A-1090 Vienna, Austria

3 Department of Physics, University of Helsinki, P.O. Box 43, FI-00014, Helsinki, Finland

4 School of Computing and Engineering, University of Huddersfield, Huddersfield HD1 3DH, U.K.

5 School of Materials, University of Manchester, Manchester M13 3PL, U.K.

6 Department of Physics and Energy, University of Limerick, Limerick, Ireland

Developing effective practical means of modifying the carrier concentration in graphene in order to create a true p- or n-doped material would represent an essential step towards graphene-based nanoelectronics. It was recently shown that this goal could be achieved by means of low energy ion implantation, a technique with the great advantage over more conventional chemical routes that it is compatible with current technology for integrated circuit fabrication. STEM-EELS results demonstrate unambiguously that $\mathrm{N}$ (for $\mathrm{n}$-doping) and $\mathrm{B}$ (for p-doping) ions were successfully implanted into the graphene sheet as pure single substitutional defects, with retention rates consistent with theoretical predictions [2]. The use of a low energy ion source was crucial in avoiding the creation of structural defects by the ejection of carbon atoms under the ion beam, although these combinations of 5-, 7- or 8member $\mathrm{C}$ rings are themselves potentially electronically active. Characterising their formation, using a far more energetic ion irradiation, is therefore essential. A combination of Raman spectroscopy and in situ electron diffraction shows that the disappearance of wrinkles in few-layer graphene sheets coincides with a progressive amorphisation of the graphene under ion irradiation [3]. High-resolution HAADF STEM imaging at $60 \mathrm{kV}$ reveals indeed that typical arrangements of defected carbon rings are present in samples that have been exposed to a high enough ion dose, while no such topological defect can be observed below a critical dose: fig. 1 .

These 'gentle' STEM observation conditions can nevertheless be used to drive the diffusion of substitutional dopants through single layer graphene, one atomic jump at a time [3]. A combined experimental and theoretical study, making use of $a b$ initio molecular dynamic calculations, reveals that for Si dopants these jumps are not due to impact on the Si atom, but to sub-threshold impact events on the surrounding $\mathrm{C}$ atoms: fig. 2. Similar events were also demonstrated to lead to the transformation of trivalently bonded $\mathrm{Si}$ dopants into the tetravalently bonded configuration, both of which have been fingerprinted by STEM-EELS [4].

Even though these results represent great strides towards nano-engineering defects in graphene, a full control over the density and nature of the defects is still difficult to achieve. By contrast, the unique structure of graphene nano-cones dictates the presence of a well-defined number of pentagonal ring defects at their tip and thus offers an ideal test material to study the impact of these defects on the electronic structure of graphene. Momentum-resolved EELS experiments in the STEM show in particular that a high enough pentagon density can lead to the confinement of plasmon modes at the apex of the cones [5], while additional interband states are created in the vicinity of the tip [6]. 


\section{References}

[1] U. Bangert, W. Pierce, Q.M. Ramasse et al., Nano Letters 13 (2013), pp. 4902-4907.

[2] C.-T. Pan, J.A. Hinks, Q.M. Ramasse, G. Greaves, U. Bangert, S.E. Donnelly and S.J. Haigh, Submitted (2014).

[3] T. Susi, J. Kotakoski, D. Kepaptsoglou, C. Mangler, T.C. Lovejoy, O.L. Krivanek, R. Zan, U. Bangert, P. Ayala, J.C. Meyer and Q.M. Ramasse, Submitted (2014).

[4] Q.M. Ramasse, C.R. Seabourne, D.M. Kepaptsoglou et al. Nano Letters 13 (2013), 4989-4985. [5]

F.S. Hage, Q.M. Ramasse, D.M. Kepaptsoglou et al., Physical Review B 88 (2013), 155408.

[6] F.S. Hage, D.M. Kepaptsoglou, C.R. Seabourne et al., Nanoscale 6 (2014), p. 1833-1839.

[7] SuperSTEM is funded by the UK Engineering and Physical Sciences Research Council (EPSRC).
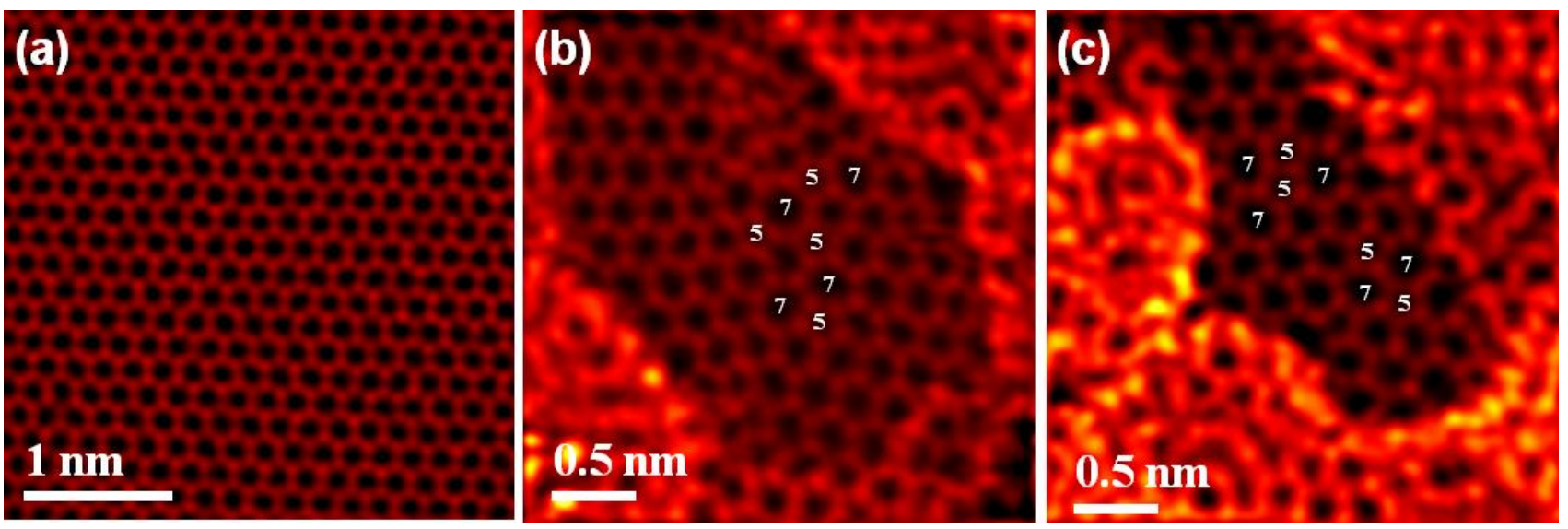

Figure 1. HAADF images of single layer graphene irradiated with $30 \mathrm{keV}$ He ions at fluences of $(a)$ $3.0 \times 10^{15}$ ions.cm ${ }^{-2}$ and $(b-c) 9.6 \times 10^{15}$ ions.cm ${ }^{-2}$ While no topological defect was observed at low ion fluences, a Haeckelite structure and an inverse Stones-Thrower-Wales defect can be seen in (b) and (c) respectively. The images were low-pass filtered for clarity. [2]
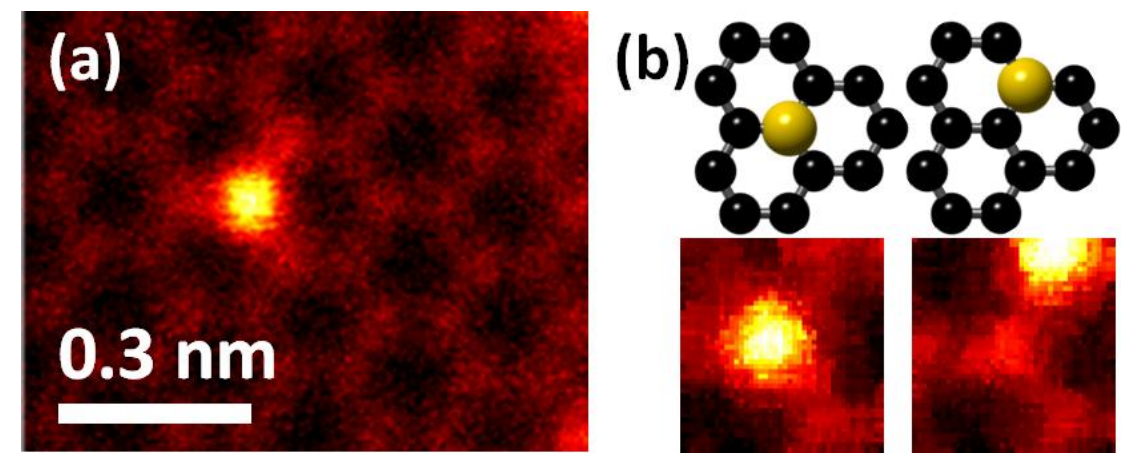

Figure 2. (a) HAADF image of a single Si substitutional dopant in trivalent configuration (fingerprinted with EELS). (b) Sequential MAADF images of a similar Si defect, showing a jump to a neighbouring atomic site: 6 consecutive frames from the original high frame rate movie are averaged (and filtered using a $5 \times 5$ Kuwahara filter for clarity), such that the two images presented here were effectively recorded $\sim 0.45 \mathrm{~s}$ apart A ball-and-stick model illustrates the atomic jump. [3] 